\title{
Long term outcome after out-of-hospital cardiac arrest with physician staffed emergency medical services: the Utstein style applied to a midsized urban/suburban area
}

\author{
B W Böttiger, C Grabner, H Bauer, C Bode, T Weber, J Motsch, E Martin
}

Department of
Anaesthesiology,
University of
Heidelberg, Im
Neuenheimer Feld 110,
D-69120 Heidelberg,
Germany
B W Böttiger
C Grabner
H Bauer
J Motsch
E Martin
Department of
Internal Medicine,
University of
Heidelberg, D-69120
Heidelberg, Germany
C Bode

Department of Surgery, University of Heidelberg, D-69120 Heidelberg, Germany $\mathrm{T}$ Weber

This paper is dedicated to Professor R Juchems on the occasion of his 70th birthday

Correspondence to: Dr Böttiger.

email: Bernd_Boettiger@ med.uni-heidelberg.de

Accepted for publication 6 July 1999

\begin{abstract}
Objective-To test the effect of a physician staffed advanced cardiac life support (ALS) system on patient outcome following out-of-hospital cardiac arrest.

Design-Observational study.

Setting-Two tier basic life support (BLS) and physician staffed ALS services in the midsized urban/suburban area of Heidelberg, Germany.
\end{abstract}

Patients-All patients suffering out-ofhospital cardiac arrest of cardiac aetiology between January 1992 and December 1994 and who were covered by ALS services.

Interventions-Physician staffed ALS services.

Main outcome measures-Return of spontaneous circulation, hospital discharge, and one year survival, according to the Utstein style.

Results-Of 330000 inhabitants, 755 suffered from cardiac arrest covered by the Heidelberg ALS services. In 512 patients, cardiopulmonary resuscitation had been initiated. Of 338 patients with cardiac aetiology, return of spontaneous circulation was achieved in 164 patients (49\%), 48 (14\%) were discharged alive, and $40(12 \%)$ were alive one year later; most of these patients showed good neurological outcome. Thus, 4.85 patients with cardiac aetiology were saved by the ALS services and discharged alive per 100000 inhabitants a year. Ventricular fibrillation or tachycardia was detected in 106 patients (31\%), other cardiac rhythms in 40 (12\%), and asystole in 192 (57\%). Hospital discharge rates (and one year survival) in these subgroups were $34.0 \% \quad(29.2 \%)$, $12.5 \%(7.5 \%)$, and $3.6 \%(3.1 \%)$, respectively. Discharge rates increased if cardiac arrest was witnessed (bystander, 20.0\%; BLS/ALS personnel, 21.4\%; nonwitnessed arrest, $3.3 \%$; $<<0.01$ ), and if the time period between the alarm and the arrival of the ALS unit was four minutes or less ( $\leqslant 4$ minutes, $30.6 \%$; 4-8 minutes, $10.4 \%$; > 8 minutes, $8.1 \%$; $p<0.001$ ). In 69 patients with bystander witnessed cardiac arrest with ventricular fibrillation, the discharge rate was $37.7 \% ; 21$ patients were alive after one year.

Conclusions-A two tier BLS and physician staffed ALS system is associated with good long term outcome of patients suffering from out-of-hospital cardiac arrest of cardiac aetiology in a midsized urban/suburban area. Further studies, however, are required to assess whether having a physician in the ALS unit is an independent determinant for improved long term outcome.

(Heart 1999;82:674-679)

Keywords: out-of-hospital cardiac arrest; emergency medical services; long term outcome; Utstein style

Patient outcome after out-of-hospital cardiac arrest depends on individual, demographic, sociological, and logistic factors. ${ }^{1-5}$ The availability, organisation, and personnel of the advanced cardiac life support (ALS) services is thought to be a crucial factor influencing the effectiveness of out-of-hospital cardiopulmonary resuscitation (CPR). ${ }^{3}$ Nationwide physician staffed ALS services, however, are cost intensive and, therefore, still a matter of debate.

Despite the large amount of data focusing on outcome after out-of-hospital cardiac arrest, little information is available on the most effective organisation of emergency medical service systems. ${ }^{3}$ Indeed, a comparison of different emergency medical service systems had previously been rendered almost impossible by the lack of consensus and uniformity in basic data evaluation, with reported hospital discharge rates of patients ranging widely between $2 \%$ and $26 \%{ }^{3}$ One of the most important reasons for these major variations is the different terminology used for data evaluation. ${ }^{2}$ This problem led the Utstein consensus conference (involving the American Heart Association, the Heart and Stroke Foundation of Canada, the Australian Resuscitation Council, and the European Resuscitation Council) to establish uniform terms and recommendations for the evaluation and reporting of data from out-ofhospital cardiac arrest. The results of this, the Utstein style, may for the first time allow a comparative analysis between different emergency medical service systems..$^{6-8}$

We investigated the effects of using a two tier emergency medical service system consisting of basic emergency medical technician (EMT) units providing basic life support (BLS) and a physician staffed ALS unit on long term patient outcome. Therefore, data from patients suffering from out-of-hospital cardiac arrest were analysed according to the guidelines of the Utstein consensus conference. ${ }^{6-8}$ 
Patients and methods

PATIENTS AND SETTING

With institutional approval, all patients who suffered an out-of-hospital cardiac arrest of cardiac aetiology between 1 January 1992 and 31 December 1994 (36 months) and who were covered by the Heidelberg ALS services were enrolled. The Heidelberg emergency medical service system serves the city of Heidelberg, a midsized urban community and its suburban environs with 21 communities, thereby covering a population of approximately 330000 residents in a region of 430 square kilometres. At the time of the study, $48 \%$ of residents were male, and $14 \%$ of residents were aged 65 years or more.

SYSTEM CONFIGURATION

During the study period, there were two components in the system, namely, the EMT ambulance crews, and one physician staffed ALS unit. Both components of this two tier system were equipped with separate vehicles and met at the scene of the arrest. The ALS unit consisted of one EMT and one physician, both based at the university hospital, who responded in a non-transport vehicle. All physicians were staff members of the departments of anaesthesiology and surgery, University of Heidelberg, Germany, who had a minimum of two years' postgraduate clinical experience and had undergone special ALS training. EMT ambulances were manned with two EMT staff. The EMT personnel underwent a minimum of six months' education in emergency medicine including BLS training and defibrillation. All EMT-BLS units were equipped with a defibrillator, and the EMT personnel were allowed to defibrillate once if they had diagnosed ventricular fibrillation (VF) in a patient suffering from cardiac arrest. During the study, six EMT-BLS units were on duty in the daytime, while four served at night. Diagnosis of cardiac arrest and CPR were performed according to the current guidelines and recommendations of the American Heart Association ${ }^{9}$ and the European Resuscitation Council. ${ }^{10}$

\section{DISPATCHING SYSTEM}

All Heidelberg emergency medical services are dispatched by one centre with a central emergency telephone system. The centre dispatches about 60000 medical calls annually, resulting in the transport of 52000 patients to five receiving hospitals. During the study, the operator at the centre decided on the basis of formal protocols whether ALS services were required. Indications for dispatching ALS services were cardiac arrest, severe trauma, poisoning, traffic accidents involving children, and further severe, acute diseases. If ALS services were required, the closest available EMTBLS unit and the physician staffed ALS unit were both dispatched simultaneously. If the EMT-BLS unit was the first vehicle to arrive on the scene, BLS was provided until the ALS unit arrived. Overall, the Heidelberg ALS unit is dispatched about 2000 times annually.

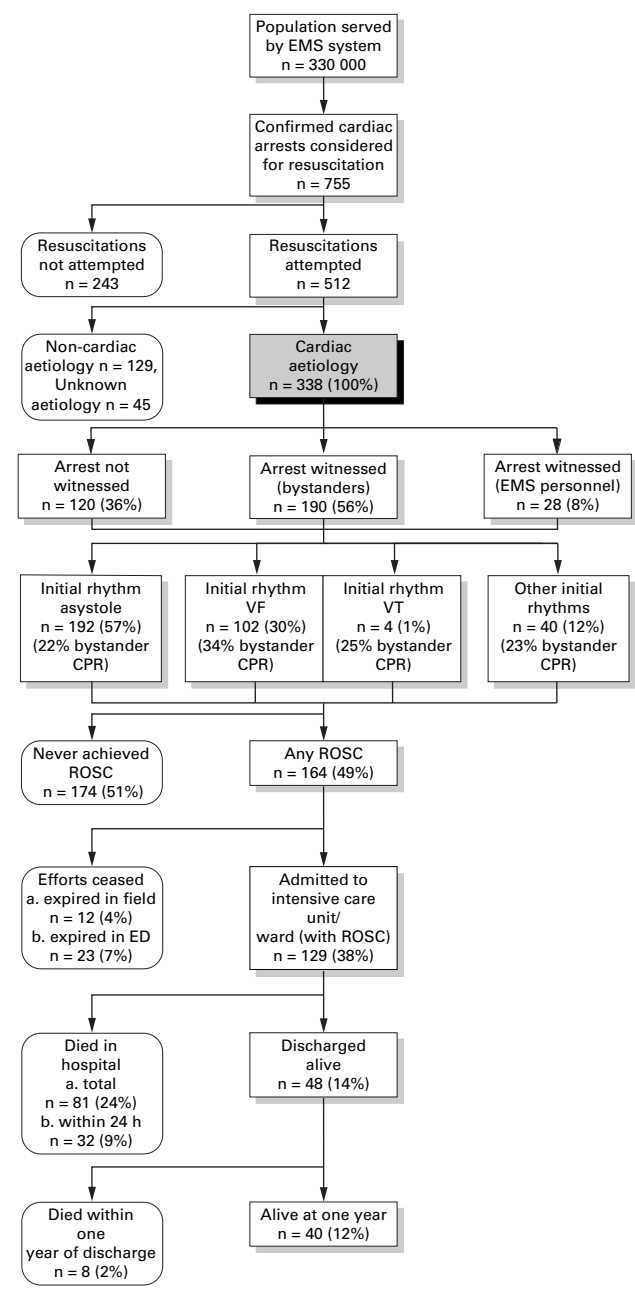

Figure 1 The Utstein template, incorporating data obtained from a two tier BLS and physician staffed ALS system in the midsized urban/suburban area of Heidelberg, Germany. CPR, cardiopulmonary resuscitation; ED, emergency department; EMS, emergency medical services; ROSC, return of spontaneous circulation; $V F$, ventricular fibrillation, VT, ventricular tachycardia.

\section{UTSTEIN STYLE}

Using the Utstein template (fig 1), ${ }^{6-8}$ information was collected on: whether the arrest was witnessed and, if so, by whom (bystander or emergency medical service personnel); who initiated CPR (bystander, regardless of the quality of bystander CPR, or emergency medical service personnel); initial rhythm (VF, pulseless ventricular tachycardia (VT), other cardiac rhythm, or asystole); whether there was a return of spontaneous circulation; and whether the patient survived to discharge and one year after discharge. ${ }^{6-8}$ To compare different emergency medical service systems, the Utstein style recommended the use of outcome data for patients whose cardiac arrest was witnessed by a bystander and who were found with VF of cardiac aetiology. ${ }^{6-8}$

\section{DATA COLLECTION}

All data were gathered using detailed protocols for data collection by the ALS physician in the prehospital setting, patient hospital records, and telephone interviews with the patient, the patient's relatives, and the patient's general practitioner. A presumed aetiology of cardiac 
Table 1 Patients'overall and cerebral outcome

\begin{tabular}{clll}
\hline & $\begin{array}{l}\text { Before cardiac arrest } \\
n(\%)\end{array}$ & $\begin{array}{l}\text { Hospital discharge } \\
n(\%)\end{array}$ & $\begin{array}{l}\text { One year after hospital } \\
\text { discharge } n(\%)\end{array}$ \\
\hline OPC & $120(35.5)$ & $11(22.9)$ & $20(50.0)$ \\
OPC1 & $136(40.2)$ & $19(39.6)$ & $10(25.0)$ \\
OPC2 & $82(24.3)$ & $16(33.3)$ & $9(22.5)$ \\
OPC3 & - & $2(4.2)$ & $1(2.5)$ \\
OPC4 & $338(100.0)$ & $48(100.0)$ & $40(100.0)$ \\
Overall & & & \\
CPC & $295(87.3)$ & $34(70.8)$ & $27(67.5)$ \\
CPC1 & $28(8.3)$ & $5(10.4)$ & $7(17.5)$ \\
CPC2 & $15(4.4)$ & $7(14.6)$ & $5(12.5)$ \\
CPC3 & - & $2(4.2)$ & $1(2.5)$ \\
CPC4 & $338(100.0)$ & $48(100.0)$ & $40(100.0)$ \\
Overall & & & \\
\hline
\end{tabular}

Overall (OPC) and cerebral (CPC) performance categories by Glasgow-Pittsburgh outcome categories $^{6-8} 1112$ of all patients suffering from out-of-hospital cardiac arrest of cardiac aetiology. OPC and CPC were assessed for the period immediately before cardiac arrest, at hospital discharge, and one year after hospital discharge. OPC1, good overall performance; OPC2, moderate disability; OPC3, severe disability; OPC4, coma or vegetative state; CPC1, good cerebral performance; CPC2, moderate cerebral disability; CPC3, severe cerebral disability; CPC4, coma or vegetative state.

arrest was determined from all available information including death certificates and necropsy reports. The outcome of all discharged patients was assessed according to the Glasgow-Pittsburgh outcome categories..$^{6-811} 12$ Overall performance categories and cerebral performance categories (table 1$)^{6-81112}$ were assessed before cardiac arrest, at hospital discharge, and one year later. Response time intervals - that is, the time intervals between the alarm and arrival of ALS on the scenewere taken from the computerised dispatch records. All data were recorded using 96 item data collection software with a closed question format. All data were double checked at data entry. Data analyses and telephone interviews were performed between July 1994 and January 1996 . The primary end points of the study were death, discharge alive, alive at one year after discharge, and overall and cerebral performance categories ${ }^{6-81112}$ at discharge and one year later.

DATA ANALYSIS AND COMPARISON WITH OTHER EMERGENCY MEDICAL SERVICE SYSTEMS

Frequencies were calculated using the total number of cardiac arrests of cardiac aetiology as the denominator. Data are expressed as mean (SD) if normally distributed (tested by Kolmogorov-Smirnov algorithms); median with range was used if data were not normally distributed. Statistical analyses were performed using $t$ test, analysis of variance (ANOVA), Scheffé test, Fisher's exact test, and $\chi^{2}$ test with Yates' correction, depending on the data configuration. Outcome data from the present study were compared with corresponding data obtained from other studies (only studies with data presentation according to the Utstein style were taken for this comparative analysis) by the $\chi^{2}$ test with Yates' correction. A value of $\mathrm{p}<0.05$ was considered significant.
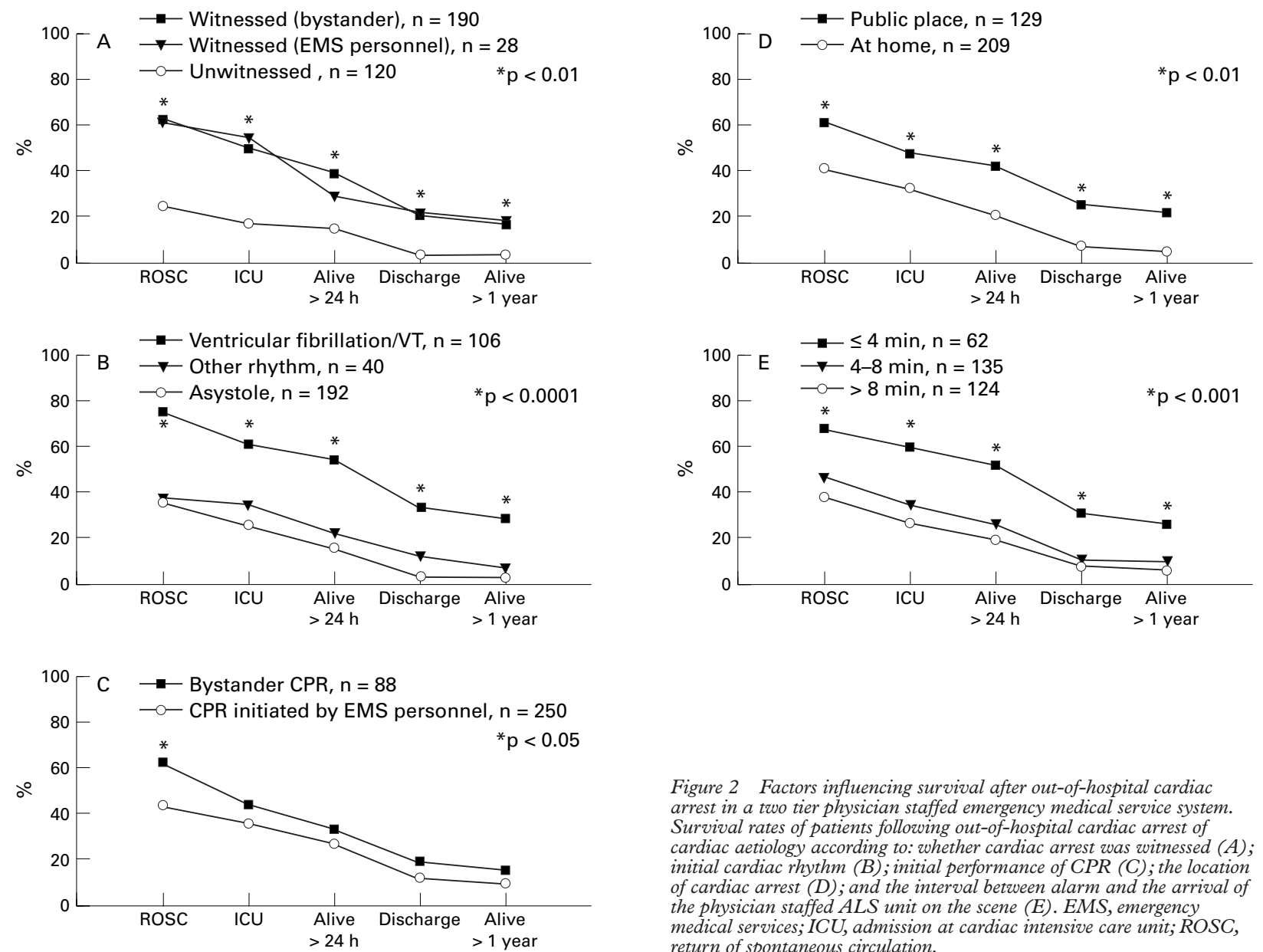

Figure 2 Factors influencing survival after out-of-hospital cardiac arrest in a two tier physician staffed emergency medical service system. Survival rates of patients following out-of-hospital cardiac arrest of cardiac aetiology according to: whether cardiac arrest was witnessed $(A)$, initial cardiac rhythm (B); initial performance of $C P R(C)$; the location of cardiac arrest (D); and the interval between alarm and the arrival of the physician staffed ALS unit on the scene (E). EMS, emergency medical services; ICU, admission at cardiac intensive care unit; ROSC, return of spontaneous circulation. 


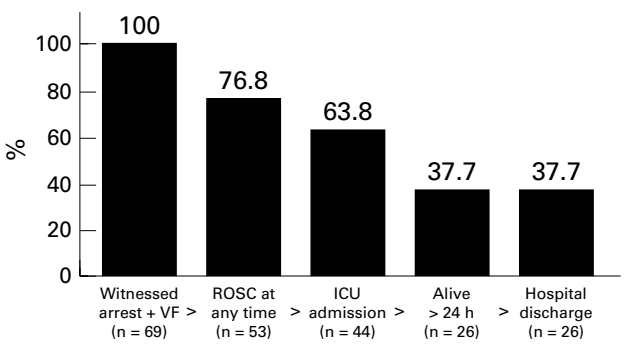

Figure 3 Outcome data for comparison of different emergency medical services. Survival rates of patients following out-of-hospital cardiac arrest of cardiac aetiology with bystander witnessed cardiac arrest and initial VF according to the Utstein recommendations. ICU, intensive care unit; ROSC, return of spontaneous circulation.

\section{Results}

Within the 36 month study period, 755 patients suffering from cardiac arrest were covered by the Heidelberg ALS services. In 243 of these patients, CPR was not attempted because death was confirmed by post mortem lividity and rigor mortis, or because cardiac arrest had obviously occurred after end stage malignant and other severe chronic diseases. In 512 patients, CPR was initiated (incidence of 51.7 CPR attempts per 100000 per year). Noncardiac aetiology was presumed in 129 patients; in 45 patients, no aetiology could be determined; 338 patients suffered from cardiac arrest of cardiac aetiology (fig 1). None of these patients was excluded or lost to follow up. The mean age of this cohort was 67 (12) years; $70.1 \%$ of these patients were male and $29.9 \%$ were female (age 65 (12) years and 71 (12) years, respectively; $\mathrm{p}<0.0001)$. Of 338 patients with an arrest of cardiac aetiology $(100 \%)$, return of spontaneous circulation could be achieved in 164 (49\%), 129 patients $(38 \%)$ were admitted alive to a cardiac intensive care unit, 48 patients $(14 \%)$ were discharged alive, and 40 patients (12\%) were alive one year after discharge (fig 1). Thus, 4.85 lives of patients with cardiac aetiology were saved by the ALS services and discharged alive per 100000 inhabitants a year. Most patients were in good condition at hospital discharge and one year later (table 1).

The collapse was witnessed in $56 \%$ of cases by bystanders and in $8 \%$ of cases by emergency medical service personnel. Both of these subgroups had an increased survival rate (discharge rates, $20.0 \%$ and $21.4 \%$, respectively) compared with patients suffering from unwitnessed cardiac arrest (discharge rate $3.3 \%$, fig 2A). Overall, 102 $(30 \%)$ patients were found with VF, four $(1 \%)$ with VT, $40(12 \%)$ with other cardiac rhythms, and 192 (57\%) with asystole (fig 2B). Hospital discharge rates (and one year survival after discharge) amounted to $34.0 \%(29.2 \%)$ of patients with initial rhythm VF/VT, $12.5 \%$ $(7.5 \%)$ of patients with other initial rhythms, and $3.6 \%(3.1 \%)$ of patients with asystole (fig 2B). Bystander CPR was initiated in $26 \%$ of patients, while CPR was initiated by emergency medical service personnel in $74 \%$ (52.2\% of these by EMT, $47.8 \%$ by the ALS unit). Of all patients with bystander CPR, $19.3 \%$ survived to hospital discharge and $15.9 \%$ one year after discharge, while among those without bystander CPR, 12.4\% survived to hospital discharge and $10.4 \%$ one year later $(p=0.09$ one year after discharge; fig $2 \mathrm{C}$ ). We observed a difference in the rate of VF $(39.8 \% v 26.8 \%$; $<<0.05)$ and in sex between patients with (male $61.4 \%$, female $38.6 \%$ ) and those without (male $73.2 \%$, female $26.8 \% ; \mathrm{p}=0.05)$ bystander CPR. The collapse occurred in $61.8 \%$ of cases at home and in $38.2 \%$ outside the home (public places). Cardiac arrest was witnessed more often if it occurred in public places $(79.8 \% v 55.0 \%$ at home; $\mathrm{p}<0.05)$, and bystander CPR was initiated significantly more often in the case of cardiac arrest in a public place $(35.5 \%$ v $20.1 \%$ at home; $\mathrm{p}<0.05)$. Patients who experienced their cardiac arrest in a public place showed significantly increased survival rates (fig $2 \mathrm{D}$ ). The response time interval of the ALS unit was

Table 2 Analysis of different emergency medical service systems

\begin{tabular}{|c|c|c|c|c|c|}
\hline Study & $\begin{array}{l}\text { Emergency medical service } \\
\text { system }\end{array}$ & $\begin{array}{l}\text { Mean response } \\
\text { interval (min) t call } \\
\text { to } B L S(A L S)\end{array}$ & $\begin{array}{l}\text { Incidence of } \\
\text { out-of-hospital CPR } \\
\text { (per } 100000 \\
\text { inhabitants per year) }\end{array}$ & $\begin{array}{l}\text { Discharge rate after bystander } \\
\text { witnessed cardiac arrest of } \\
\text { cardiac aetiology with initial } \\
\text { rhythm VF } n(\%)\end{array}$ & $\begin{array}{l}\text { Discharge rate after cardiac } \\
\text { arrest of cardiac aetiology } \\
n(\%)\end{array}$ \\
\hline \multicolumn{6}{|c|}{ EMT-BLS and physician staffed ALS services } \\
\hline Heidelberg 1999 & BLS + ALS-P & $(8.0) \ddagger$ & 34.24 & $26 / 69(38)$ & $48 / 338(14)$ \\
\hline Bonn $1997^{13}$ & BLS + ALS-P & $5.0(8.2)$ & 48.33 & $41 / 118$ (35) NS & $74 / 464(16) \mathrm{NS}$ \\
\hline Helsinki $1996^{14}$ & $\mathrm{BLS}+\mathrm{ALS}+\mathrm{ALS}-\mathrm{P}$ & $7.0(10.3)$ & 53.07 & $41 / 126$ (33) NS & $44 / 255$ (17) NS \\
\hline Mainz $1994^{15}$ & BLS + ALS-P & $6.0(10.0)$ & 78.14 & $13 / 54(24) \mathrm{NS}$ & $19 / 211(9) \mathrm{NS}$ \\
\hline Helsinki $1990^{16}$ & BLS + ALS-P & $5.5(10.7) \ddagger$ & 48.20 & $39 / 144$ (27) NS & ND \\
\hline \multicolumn{6}{|c|}{ EMT-BLS and/or ALS services without physicians } \\
\hline Auckland $1995^{19}$ & ALS & $(7.0)$ & 38.11 & $89 / 545(16)^{\star \star}$ & $135 / 1069$ (13) NS \\
\hline Oakland County $1995^{25}$ & $\mathrm{BLS}+\mathrm{ALS}$ & $3.6(6.5)$ & 53.80 & $72 / 476(15)^{\star \star}$ & ND \\
\hline York-Adams $1994^{21}$ & $\mathrm{BLS}+\mathrm{ALS}$ & ND & 73.05 & $22 / 260(8)^{\star \star}$ & $36 / 599(6)^{\star \star}$ \\
\hline New York City $1994^{5}$ & $\mathrm{BLS}+\mathrm{ALS}$ & $9.4(13.0)$ & 63.52 & $22 / 415(5)^{\star \star}$ & $33 / 2329(1)^{\star \star}$ \\
\hline Scotland $1993^{22}$ & BLS & 9.0 & 32.85 & $71 / 643(11)^{\star \star}$ & $174 / 1676(10)^{\star}$ \\
\hline Tucson $1992^{26}$ & BLS + ALS & $4.1(5.1)$ & 50.50 & $18 / 118(15)^{\star \star}$ & $22 / 372(6)^{\star \star}$ \\
\hline Chicago $1991^{18}$ & ALS & $(13.0)$ & 107.37 & $15 / 371(4)^{\star \star}$ & $55 / 3221(2)^{\star \star}$ \\
\hline Tucson $1990^{23}$ & BLS + ALS & $3.0(5.0)$ & 62.08 & $21 / 96(22)^{\star}$ & $25 / 298(8)^{\star}$ \\
\hline York-Adams $1988^{20}$ & $\mathrm{BLS}+\mathrm{ALS}$ & ND & 47.11 & $47 / 382(12)^{\star \star}$ & $68 / 1066(6)^{\star \star}$ \\
\hline Milwaukee $1986^{24}$ & $\mathrm{BLS}+\mathrm{ALS}$ & $2.0(5.0)$ & ND & $183 / 779(23)^{\star}$ & ND \\
\hline King County $1985^{17}$ & $\mathrm{BLS}+\mathrm{ALS}$ & $4.4(9.2)$ & 38.91 & $314 / 951$ (33) NS & ND \\
\hline
\end{tabular}

Register of studies in patients suffering from out-of-hospital cardiac arrest of cardiac aetiology and reporting their data according to the Utstein recommendations. Statistical analysis $\left(\chi^{2}\right.$ test) revealed that outcome in the present study is comparable to that of all other two ${ }^{13}{ }^{15}$ and three $^{14}$ tier EMT-BLS and physician staffed ALS systems and to one ALS system with paramedics, ${ }^{17}$ all located in midsized urban/suburban areas. ${ }^{13-16}$

${ }^{\star} \mathrm{p}<0.05,{ }^{\star \star} \mathrm{p}<0.001 ;$ †Time between emergency call and arrival BLS/ALS on the scene; $\ddagger$ data reflect time interval between alarm of ALS unit and arrival on the scene.

ALS, advanced cardiac life support by paramedics; ALS-P, advanced cardiac life support by physicians; BLS, basic life support; EMT-BLS, emergency medical technician service providing basic cardiac life support; ND, not determined; NS, not significant. 
inversely related to long term outcome; an interval of four minutes or less was associated with a higher long term survival rate (fig 2E).

Overall, the mean response time interval for survivors (6.1 (4.0) minutes) was shorter than that for non-survivors (8.3 (4.0) minutes; $\mathrm{p}<0.001)$. Increased age was associated with poorer prognosis (survivors, 62 (13) years, non-survivors 68 (12) years; $p<0.01$ ) in patients admitted to the intensive care unit. With regard to the duration of CPR in these patients, there was no difference between long term survivors (20 (16) minutes) and nonsurvivors (21 (14) minutes). Nine patients were stabilised and survived after more than 30 minutes of CPR.

In patients whose cardiac arrest was witnessed by a bystander and who were found with VF of cardiac aetiology ( $n=69 ; 6.97$ patients per 100000 inhabitants a year), the discharge rate was $37.7 \%(\mathrm{n}=26 ; 2.63$ patients per 100000 inhabitants a year) (fig 3); $21(30.4 \%)$ of these patients were alive at one year. When comparing the present outcome data with those of other emergency medical service systems according to the Utstein recommendations, it was found that the present survival rates are close to those obtained from all other two and three tier BLS and physician staffed ALS services ${ }^{13-16}$ and to one ALS system with paramedics, ${ }^{17}$ all located in midsized urban/suburban areas (table 2). Emergency medical service systems in large metropolitan and rural areas without physician staffed ALS services revealed lower survival rates (table 2)..$^{58-26}$

\section{Discussion}

In a midsized urban/suburban system with two tier BLS and ALS services, where ALS is provided by physicians, $14 \%$ of patients resuscitated after cardiac arrest of cardiac aetiology were discharged alive, and $12 \%$ were alive at one year after discharge. Most of these patients showed good neurological outcome. Several factors influencing outcome could be identified. In the case of VF, survival increased if the cardiac arrest was witnessed, if it occurred in a public place, and/or if the time period between the alarm being raised and the arrival of the ALS unit was four minutes or less. All these factors reflect the time delay between cardiac arrest and initiation of CPR, and it is well known that early initiation of CPR is associated with a more favourable outcome. ${ }^{17}{ }^{27} \mathrm{We}$ also recognised the effect that age has on long term survival. Age, however, must not be an independent predictor of outcome, because age may be associated with a higher incidence of unfavourable pre-arrest health conditions. ${ }^{28}$ The rate of return of spontaneous circulation increased with bystander CPR. The lack of significant differences in survival at hospital discharge and one year after discharge, however, does not indicate that bystander CPR has no beneficial effects, since there was a notable trend towards improved long term outcome.

The present data are reported according to the recommendations of the Utstein consensus conference to allow comparison of our emer- gency medical service system with others. ${ }^{6-8}$ For this purpose, the Utstein consensus conference recommended the use of outcome data obtained from patients with bystander witnessed cardiac arrest of cardiac aetiology and initial rhythm $\mathrm{VF}^{6-8}$ In the present study, $37.7 \%$ of these patients were discharged alive, and $30.4 \%$ were alive one year after discharge. The comparative analysis revealed that survival rates in the present study are similar to those from other two tier BLS and physician staffed ALS service systems in midsized urban/ suburban areas. ${ }^{13-16}$ The lowest survival rates could be observed in large metropolitan areas (table 2). ${ }^{518}$ In large metropolitan areas and in rural communities, call-response intervals often exceeded 10 minutes, ${ }^{5} 1822$ while in midsized urban/suburban areas, as in the present setting, BLS and ALS services are usually on the scene within 5-10 minutes. ${ }^{13-17}$ Moreover, subgroup analysis of survival among patients in whom cardiac arrest occurred after arrival of emergency medical service personnel (a setting where response time intervals could not affect outcome) suggests that factors such as race, socioeconomic status, substandard quality of health care for large groups of inhabitants, and lack of adequate bystander CPR may be the primary cause of higher cardiac arrest mortality in residents of larger cities and metropoli$\tan$ areas than in individuals living in more suburban or rural settings. ${ }^{4}$

However, by reviewing studies on 39 emergency medical service programmes from 29 different locations published between 1967 and 1988, Eisenberg and colleagues showed that the qualification of the personnel and the configuration of the emergency medical service system are the most important independent variables affecting outcome after out-ofhospital cardiac arrest. ${ }^{3}$ The highest survival rates could be observed in two tier BLS and paramedic staffed ALS systems. Physician staffed ALS systems were not included in this comparative analysis because of a lack of data. ${ }^{3}$ One can speculate, however, that a physician has more experience in intubation of the trachea and immediate venous access than other emergency medical service personnel, and thus a physician staffed emergency medical service system may be superior.

The present analysis may support the view that physician staffed ALS systems are associated with higher survival rates in patients suffering from out-of-hospital cardiac arrest of cardiac aetiology. All the data from physician staffed ALS systems, however, are related to midsized urban/suburban areas, ${ }^{13-16}$ while the available data from emergency medical service systems without a physician include a variety of regions, mostly large metropolitan and rural areas (table 2). ${ }^{18-26}$ It cannot be sufficiently excluded by the present analysis that socioeconomic, demographic, and racial factors may cause the observed differences. Therefore, it remains unclear whether ALS training per se combined with the availability of a defibrillator or the presence of a physician on board the ALS unit makes the major difference with regard to the outcome of cardiac arrest. 
In conclusion, the present data suggest that early initiation of CPR by bystanders and short response time intervals for physician staffed ALS units can reduce mortality in patients suffering from an out-of-hospital cardiac arrest of cardiac aetiology. A two tier BLS and physician staffed ALS system is associated with good long term outcome in patients suffering from an out-of-hospital cardiac arrest of cardiac aetiology in a midsized urban/suburban area. Further studies, however, are required to assess whether the fact that a physician is on board the ALS unit is an independent determinant for improved long term outcome. This study was supported by a grant (No. 11/94) from the
medical faculty of the University of Heidelberg, Germany, to Dr medical faculty of the University of Heidelberg, Germany, to Dr Bottiger. The authors would like to thank the emergency staff of the German Red Cross (Heidelberg), Professor C Herfarth, Dr J Stern, and A Thome (department of surgery, University of Heidelberg), Professor W Kübler (department of internal medicine, University of Heidelberg), and all colleagues involved in their kind support. In addition, our thanks go to Dr M Fischer (department of anaesthesiology, University of Bonn, Germany) for helpful suggestions in data analysis.

1 Becker LB, Han BH, Meyer PM, et al. Racial differences in the incidence of cardiac arrest and subsequent survival. $N$ Engl F Med 1993;329:600-6.

2 Eisenberg MS, Copass MK, Hallstrom AP, et al. Treatmen of out-of-hospital cardiac arrests with rapid defibrillation by emergency medical technicians. $N$ Engl $f \mathrm{Med}$ 1980;302:1379-83.

3 Eisenberg MS, Horwood BT, Cummins RO, et al. Cardiac arrest and resuscitation: a tale of 29 cities. Ann Emerg Med 1990;19:179-86.

4 Hallstrom A, Boutin P, Cobb L, et al. Socioeconomic status and prediction of ventricular fibrillation survival. $A m \mathcal{F}$ Public Health 1993;83:245-8.

5 Lombardi G, Gallagher EJ, Gennis P. Outcome of out-of-hospital cardiac arrest in New York City. The out-of-hospital cardiac arrest in New York City. The pre-hospital arrest survi

6 Chamberlain D, Cummins RO. Recommended guidelines for uniform reporting of data from out-of-hospital cardiac arrest: the 'Utstein style'. The European Resuscitation Council, American Heart Association, Heart and Stroke Foundation of Canada and Australian Resuscitation Council. Eur f Anaesthesiol 1992;9:245-56.

7 Cummins RO, Chamberlain DA, Abramson NS, et al. Recommended guidelines for uniform reporting of data from out-of-hospital cardiac arrest: the Utstein Style. A statement for health professionals from a task force of the American Heart Association, the European Resuscitation Council, the Heart and Stroke Foundation of Canada, and the Australian Resuscitation Council. Circulation 1991;84: 960-75.

8 European Resuscitation Council, American Heart Associtrone ation, Heart and Stroke Foundation of Canada, and lines for uniform reporting of data from out-of-hospital cardiac arrest (new abridged version). The "Utstein style". Br Heart f 1992;67:325-33.

9 Emergency Cardiac Care Committee and Subcommittees, American Heart Association. Guidelines for cardiopulmonary resuscitation and emergency cardiac care. $\mathcal{F} A M A$ 1992;268:2171-298.

10 European Resuscitation Council. Guidelines for basic and advanced life support. Resuscitation 1992;24:103-21.

11 Brain Resuscitation Clinical Trial I Study Group. A randomized clinical study of cardiopulmonary-cerebral resuscitation: design, methods, and patient characteristics. Am $\mathcal{F}$ Emerg Med 1986;4:72-86.

12 Jennett $\mathrm{B}$, Bond $\mathrm{M}$. Assessment of outcome after severe brain damage. Lancet 1975;i:480-4.

13 Fischer M, Fischer NJ, Schüttler J. One-year survival after out-of-hospital cardiac arrest in Bonn city: outcome report according to the 'Utstein style'. Resuscitation 1997;33:23343.

14 Kuisma M, Määttä T. Out-of-hospital cardiac arrest in Helsinki: Utstein style reporting. Heart 1996;76:18-23.

15 Schneider T, Mauer D, Diehl P, et al. Quality of on-site performance in prehospital advanced cardiac life support (ACLS). Resuscitation 1994;27:207-13.

16 Silfvast T. Prehospital resuscitation in Helsinki, Finland. Am 7 Emerg Med 1990;8:359-64.

17 Cummins RO, Eisenberg MS, Hallstrom AP, et al. Survival of out-of-hospital cardiac arrest with early initiation of cardiopulmonary resuscitation. Am f Emerg Med 1985;3:1149.

18 Becker LB, Ostrander MP, Barret J, et al. Outcome of CPR in a large metropolitan area: where are the survivors? Ann Emerg Med 1991;20:355-61.

19 Crone PD. Auckland ambulance service cardiac arrest data 1991-3. N Z Med f 1995;108:297-9.

20 Eitel DR, Walton SL, Guerci AD, et al. Out-of-hospital cardiac arrest: a six-year experience in a suburban-rural system. Ann Emerg Med 1988;17:808-12.

21 Kass LE, Eitel DR, Sabulsky NK, et al. One-year survival after prehospital cardiac arrest: the Utstein style applied to a rural-suburban system. Am f Emerg Med 1994;12:17-20.

22 Sedgwick ML, Dalziel K, Watson J, et al. Performance of an established system of first responder out-of-hospital defibrillation. The results of the second year of the Heartstart Scotland project in the 'Utstein style'. Resuscitation 1993;26:75-88.

23 Spaite DW, Hanlon T, Criss EA, et al. Prehospital cardiac arrest: the impact of witnessed collapse and bystander CPR in a metropolitan EMS system with short response times. Ann Emerg Med 1990;19:1264-9.

24 Stueven H, Troiano P, Thompson B, et al. Bystander/first responder CPR: ten years experience in a paramedic system. Ann Emerg Med 1986;15:707-10.

25 Swor RA, Jackson RE, Cynar M, et al. Bystander CPR, ventricular fibrillation, and survival in witnessed, unmonitored out-of-hospital cardiac arrest. Ann Emerg Med 1995;25: $780-4$

26 Valenzuela TD, Spaite DW, Meislin HW, et al. Case and survival definitions in out-of-hospital cardiac arrest. $\mathcal{F} A M A$ 1992;267:272-4.

27 Mullie A, Van Hoeyweghen R, Quets A. Influence of time intervals on outcome of CPR. The cerebral resuscitation study group. Resuscitation 1989;17(suppl):S199-206.

28 Juchems R, Wahlig G, Frese W. Influence of age on the survival rate of out-of-hospital and in-hospital resuscitation. Resuscitation 1993;26:23-9. 